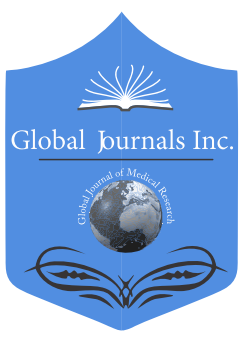

\title{
Denoising and Analysis of EMG Signal using Wavelet Transform
}

By Iffat Ara

Pabna University of Science and Technology

Abstract- EMG is the recording of the electrical activity produced within the muscle fibers. Measurement of EMG signal is corrupted by additive noise whose signal-to-noise ratio (SNR) varies. Feature extraction is an important step for EMG classification. Time domain and frequency domain parameters were chosen as representative features for EMG signals. In this thesis, the Wavelet transform and wavelet coefficients have adopted to represent the EMG signals. Wavelet transform (WT) has been applied also in this research for the analysis of the surface electromyography signal (SEMG). The properties of wavelet transform turned out to be suitable for nonstationary EMG signals. Also Spectrum analysis has been applied to various types of EMG signal.

Keywords: EMG, wavelet transform, SNR, myopathy, neuropathy.

GJMR-D Classification: NLMC Code: WN 600

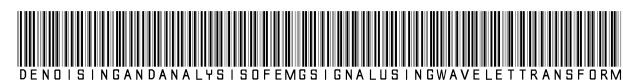

Strictly as per the compliance and regulations of:

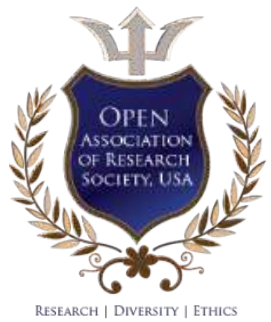

(C) 2020. Iffat Ara. This is a research/review paper, distributed under the terms of the Creative Commons AttributionNoncommercial 3.0 Unported License http://creativecommons.org/licenses/by-nc/3.0/), permitting all non-commercial use, distribution, and reproduction in any medium, provided the original work is properly cited. 


\section{Denoising and Analysis of EMG Signal using Wavelet Transform}

Iffat Ara

\begin{abstract}
EMG is the recording of the electrical activity produced within the muscle fibers. Measurement of EMG signal is corrupted by additive noise whose signal-to-noise ratio (SNR) varies. Feature extraction is an important step for EMG classification. Time domain and frequency domain parameters were chosen as representative features for EMG signals. In this thesis, the Wavelet transform and wavelet coefficients have adopted to represent the EMG signals. Wavelet transform (WT) has been applied also in this research for the analysis of the surface electromyography signal (SEMG). The properties of wavelet transform turned out to be suitable for nonstationary EMG signals. Also Spectrum analysis has been applied to various types of EMG signal.

Keywords: EMG, wavelet transform, SNR, myopathy, neuropathy.
\end{abstract}

\section{INTRODUCTION}

T$M G$ is the recording of the electrical activity produced within the muscle fibers. The nervous system controls the voluntary movement of various body parts in humans by contracting and relaxing various skeletal muscles. To instantiate a contraction, a neuron generates a small electrical potential on the surface of the muscle fiber. This electrical potential causes depolarization of the muscle fiber tissue and a following depolarization waveform. This waveform travels the length of the muscle fiber and is known as the Action Potential (AP) $[1,7]$.

To collect EMG signal, two techniques are applied, namely, Surface Electromyography (SEMG) and needle Electromyography. Although both mentioned techniques result in EMG signal, SEMG is more popular than needle EMG since SEMG is a noninvasive technique and more convenient to use [2] .

The wavelet transform, a multi-resolution timefrequency analysis, is preferred for EMG analysis. The results of this work indicated using dubecies family as mother wavelet (MW) in 8 decomposition level to determine the muscle fatigue status. Wavelet analysis has been known as a flexible technique because a vast variety of the wavelet function exists in applying wavelet techniques. Power spectrum analysis on EMG signal was applied through different MWs in this study.

Author: Department of Information and Communication Engineering, Pabna University of Science and Technology, Bangladesh.

e-mail: ara.iffat@ymail.com

\section{METHODS}

To analyze EMG signal three domains of study have been utilized in many researches, time domain, frequency domain and time frequency domain. EMG is a non-stationary signal while it is assumed as a stationary signal in time domain. Also, working in the frequency domain has the problem of not having access to time domain. Consequently, wavelet analysis has been applied as a strong and more compatible technique with the nature of EMG signal since wavelet has proved much capability to analyze biomedical signals. These SEMG signals were decomposed using DWT with db6 wavelet functions. The DWT was implemented using MATLAB [3].

The wavelet transform is a convolution of the wavelet function $\psi(\mathrm{t})$ with the signal $x(\mathrm{t})$. Orthonormal dyadic discrete wavelets are associated with scaling function $\varphi(t)$. The scaling function can be convolved with the signal to produce approximation coefficients $\mathrm{S}$. The discrete wavelet transforms (DWT) can be written as:

$$
\mathrm{T}_{\mathrm{m}, \mathrm{n}}=\int_{-\infty}^{\infty} \mathrm{x}(\mathrm{t}) \psi_{\mathrm{m}, \mathrm{n}}(\mathrm{t}) \mathrm{dt} \ldots \ldots \ldots \ldots \ldots .
$$

Where $T_{m, n}$ is known as the detail coefficient at scale and location indices $(m, n)$.

The approximation coefficients of the signal at the scale $m$ and location $n$ can be represented by:

$$
S_{m, n}=\int_{-\infty}^{\infty} x(t) \varphi_{m, n}(t) d t \ldots \ldots \ldots \ldots .
$$

A discrete approximation of the signal can be shown as

$$
\mathrm{x}_{0}(\mathrm{t})=\mathrm{x}_{\mathrm{M}}(\mathrm{t})+\sum_{\mathrm{m}=1}^{\mathrm{M}} \mathrm{d}_{\mathrm{m}}(\mathrm{t}) \ldots \ldots \ldots \ldots
$$

Where the mean signal approximation at scale $M$ is

$$
\mathrm{x}_{\mathrm{M}}(\mathrm{t})=\mathrm{S}_{\mathrm{M}, \mathrm{n}} \varphi_{\mathrm{M}, \mathrm{n}}(\mathrm{t}) \ldots \ldots \ldots \ldots \ldots \ldots \ldots \ldots \ldots \ldots \ldots \ldots \ldots
$$

The detail signal approximation corresponding to scale index $m$ is defined for a finite length signal as

$$
d_{m}(t)=\sum_{n=0}^{2 M-m} T_{m, n} \psi_{m, n}(t) \ldots \ldots \ldots
$$

The signal approximation at a specific scale was a combination of the approximation and detail at 
the next lower scale. If scale $m=3$ was chosen, it can be shown that the signal approximation is given by

$$
x_{3}(t)=x_{0}(t)-d_{1}(t)-d_{2}(t)-d_{3}(t) \ldots \ldots \ldots
$$

Corresponding to the successive stripping of high frequency information (contained within the $d_{m}(t)$ ) from the original signal at each step [4]. This is referred to as multi-resolution analysis of a signal using wavelet transform, and is the basic of our procedure.

\section{Figure 1: Shows the flow chart for the EMG feature extraction step}

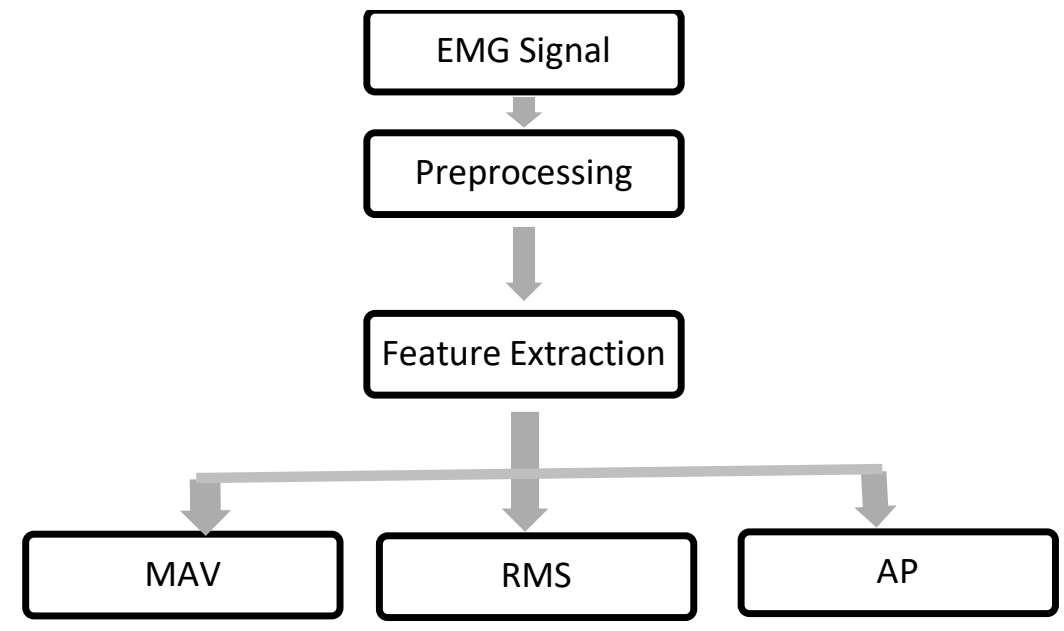

Figure 1: Flow chart of EMG features extraction algorithm

\section{a) Prototypes of Wavelet}

The large number of known wavelet families and functions provides a rich space in which to search for a wavelet which will very efficiently represent a signal of interest in a large variety of applications. Wavelet families include Biorthogonal, Coiflet, Haar, Symmlet, Daubechies wavelets [4], etc. There is no absolute way to choose a certain wavelet. The choice of the wavelet function depends on the application. The Haar wavelet algorithm has the advantage of being simple to compute and easy to understand. The Daubechies algorithm is conceptually more complex and has a slightly higher computational overhead. But, the Daubechies algorithm picks up detail that is missed by the Haar wavelet algorithm. Even if a signal is not well represented by one member of the $\mathrm{Db}$ family, it may still be efficiently represented by another. Selecting a wavelet function which closely matches the signal to be processed is of utmost importance in wavelet applications [5]. Daubechies wavelet families their energy spectrums are concentrated around low frequencies.

\section{b) Denoising Of EMG Signal}

The Surface EMG (SEMG) signals was denoised using discrete wavelet transform (DWT) and a threshold method. The DWT and threshold based denoising was implemented using MATLAB. Wavelets commonly used for denoising biomedical signals include the Daubechies (db2, db8, and db6) wavelets and orthogonal Meyer wavelet. The wavelets are generally chosen whose shapes are similar to those of the MUAP.

A process of removing the noise of a signal is called as de-noising. Once the signal is preprocessed then it can be used for further processing. In this study the identified high frequency components are D1, D2. These components must be filtered by applying a threshold. Then the threshold components are removed from the signal. The original EMG signals and the denoised EMG signal of length 800 samples are shown in Figure 2.

\section{Results}

The raw SEMG data was downloaded from Physiobank database. Any of the WFs (db2, db4, db6, and db8) are effective for noise removal in the case of SEMG. In this experiment WF db6 is chosen and found to be effective for noise removal. As known, such signal is normally a function of time and is explained in terms of amplitude, frequency and phase. Thus, power spectrum generated from output signal was examined to identify appropriate signal parameters to distinguish signal from respective patients. From power spectrum point of view, both signals can be easily analyzed and classified in terms of amplitude, in terms of power spectral density. Many parameters can be calculated to use as rule base classifier input, which is mean absolute value (MAV), Average Power (AP), amplitude in terms of root mean square (RMS), minimum and maximum power spectral density. 
The wavelet analysis tool as well as the graphical result for different normal and abnormal EMG signal is presented. The wavelet analysis of EMG signal is performed using MATLAB software. MATLAB is a high performance; interactive system which allows to solve many technical computing problem. MATLAB 7.5 versions have been used to write computer program designed for analyzing.
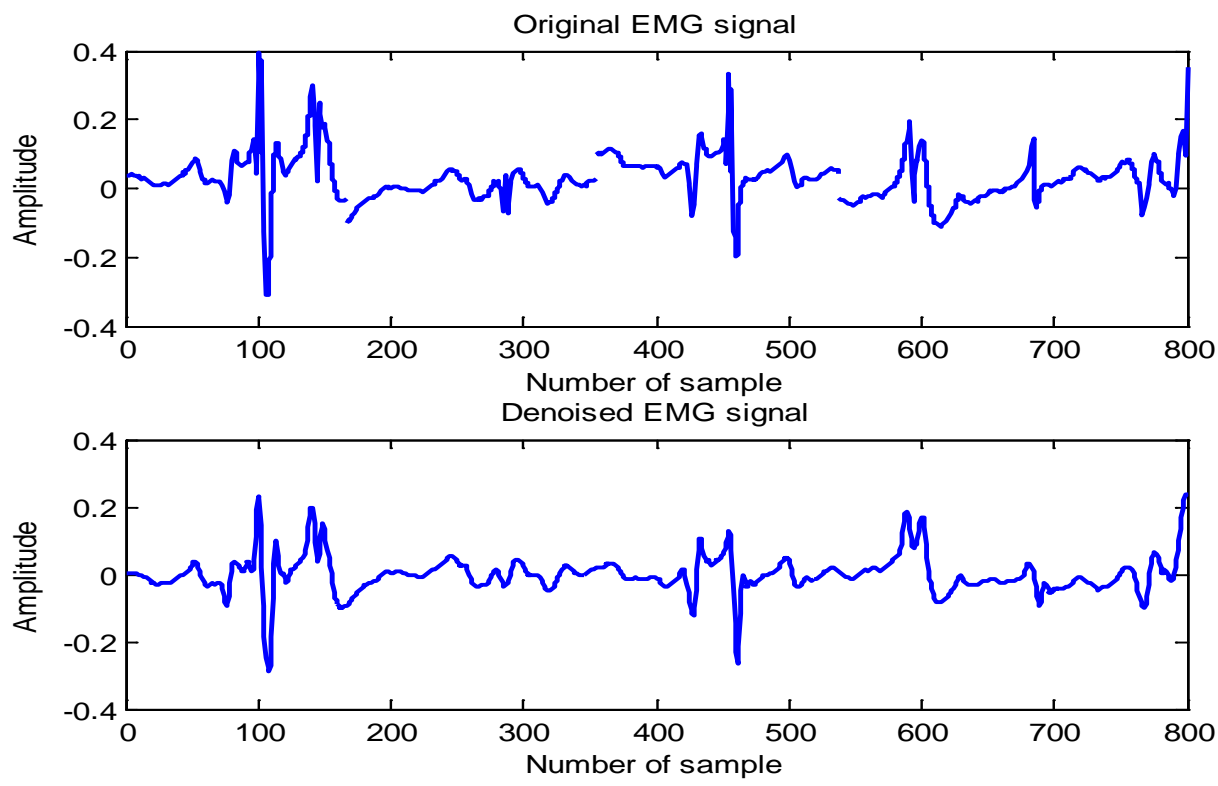

Figure 2: Original and De-noised Healthy EMG signal

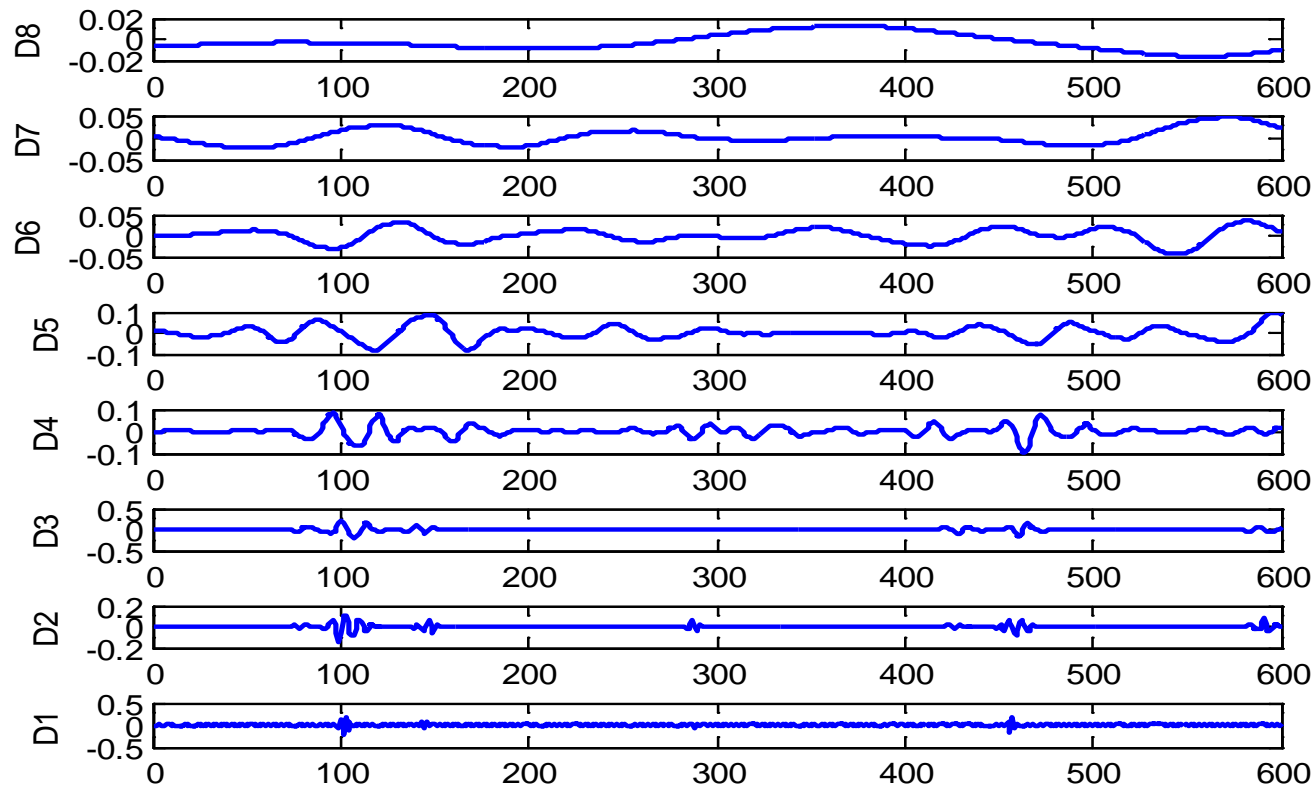

Figure 3: Representation of different level of wavelet detail coefficient 

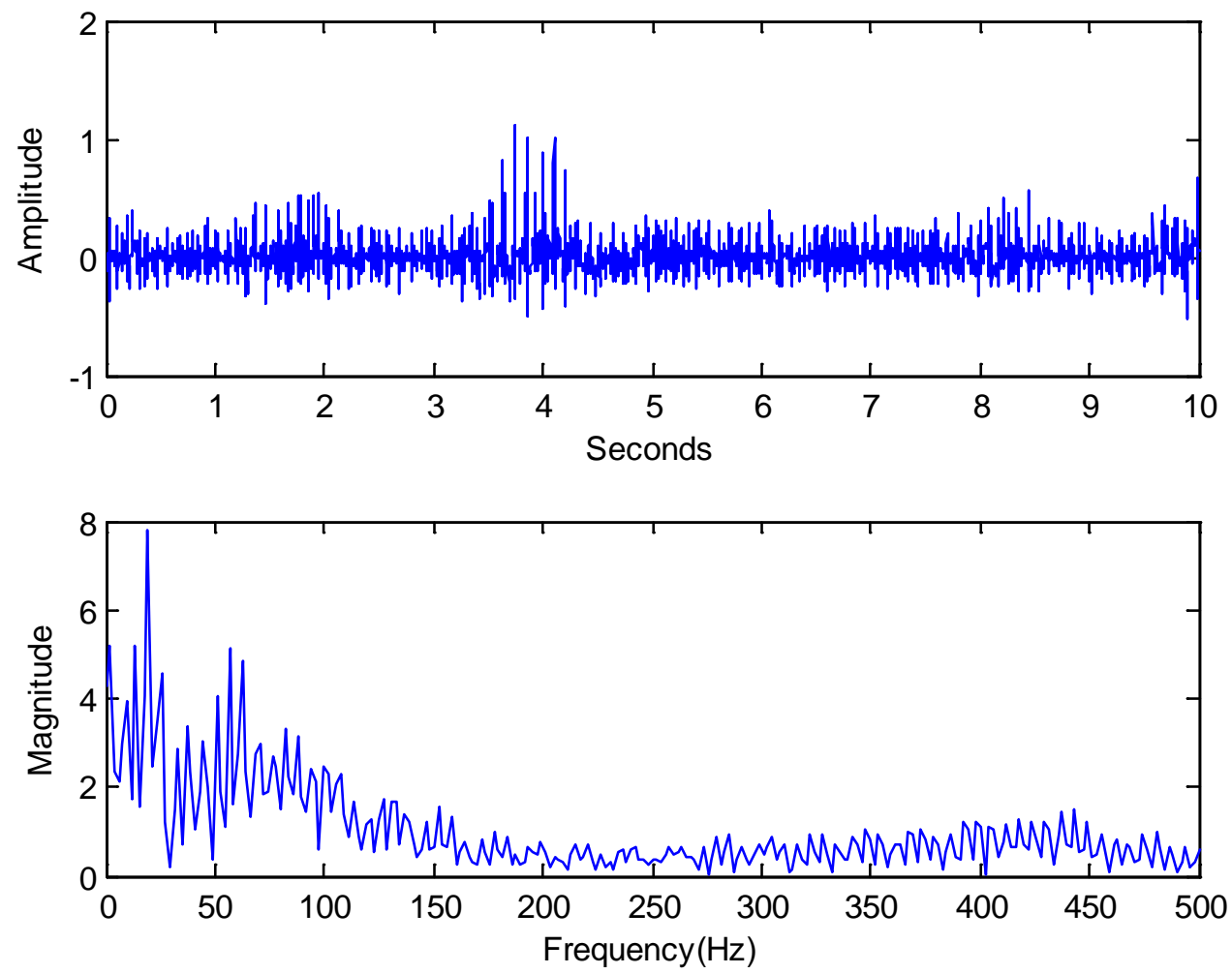

Figure 4: (a) Original healthy EMG signal and (b) Frequency domain representation of EMG

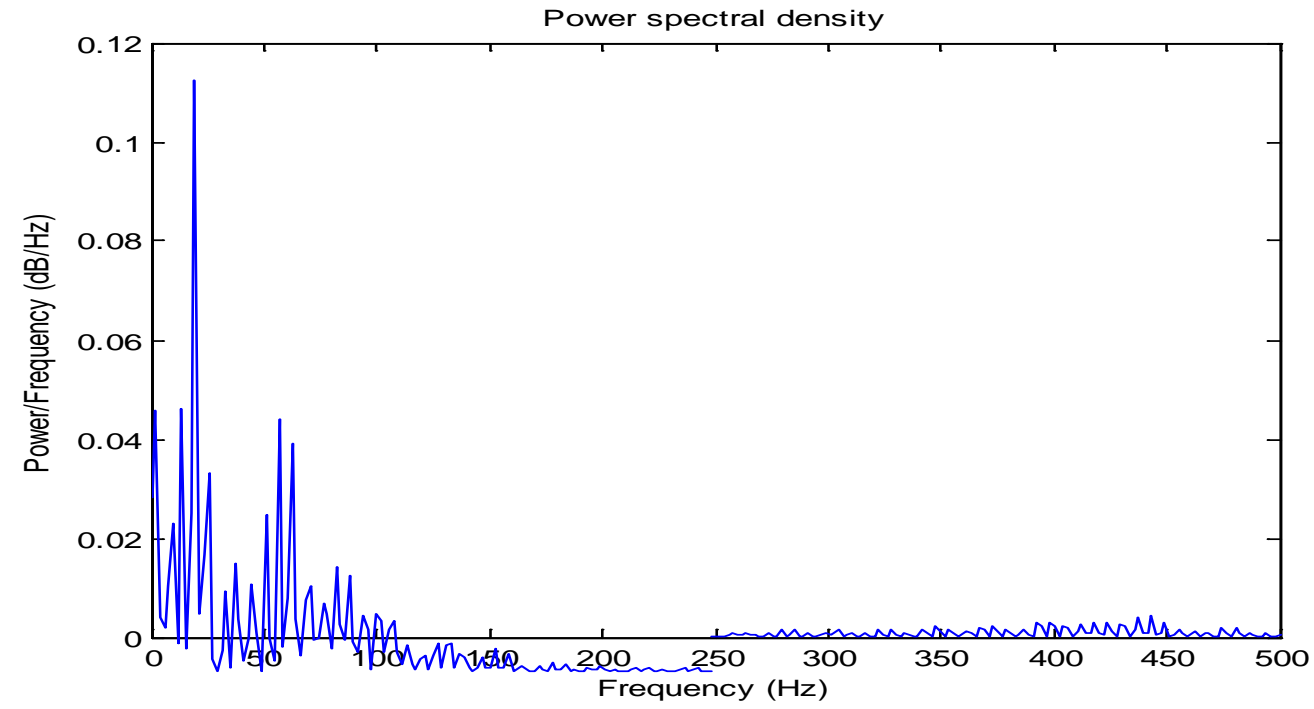

Figure 5: Power spectral density of Healthy EMG signal 

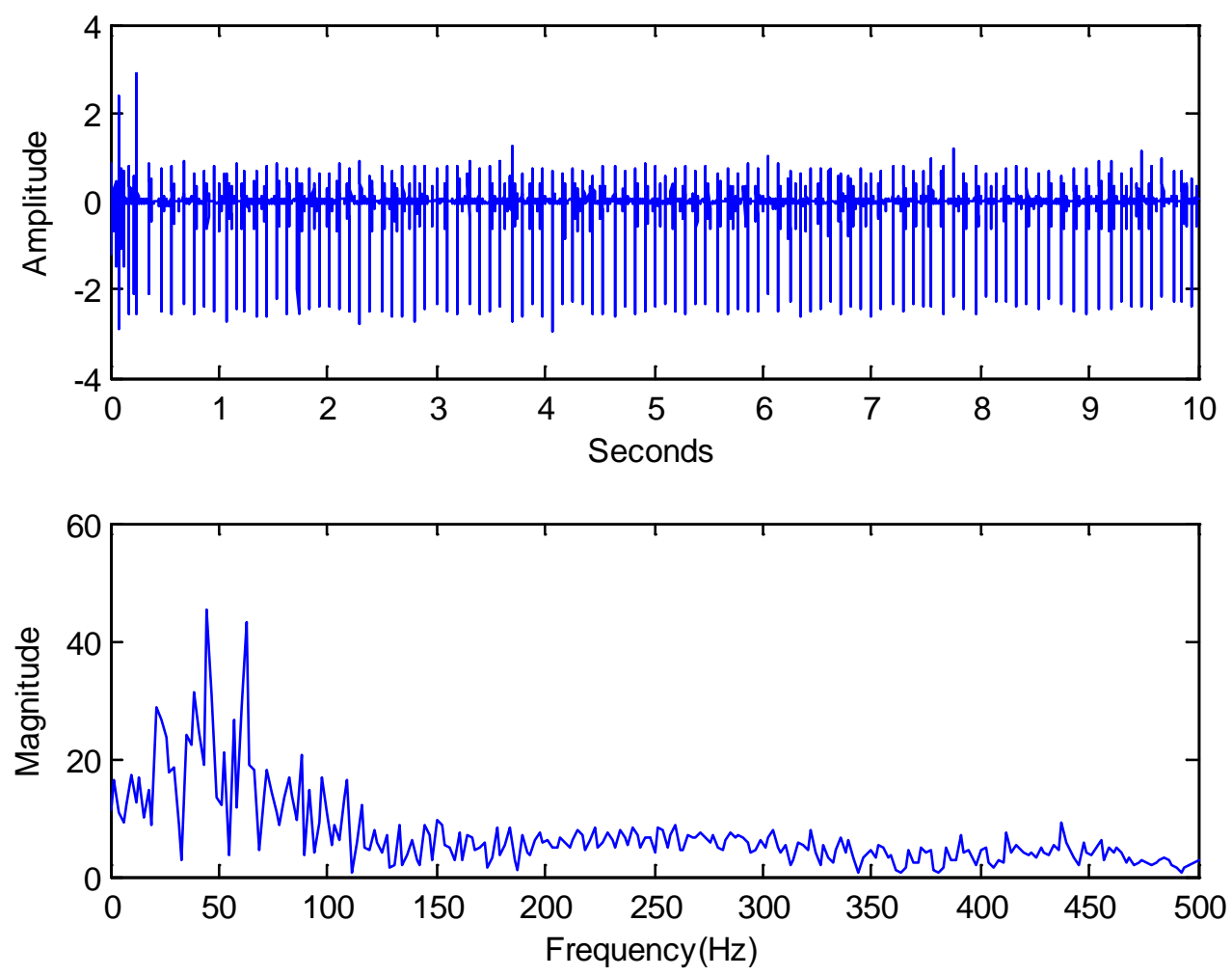

Figure 6: (a) Original Neuropathy EMG signal and (b) Frequency domain representation of EMG

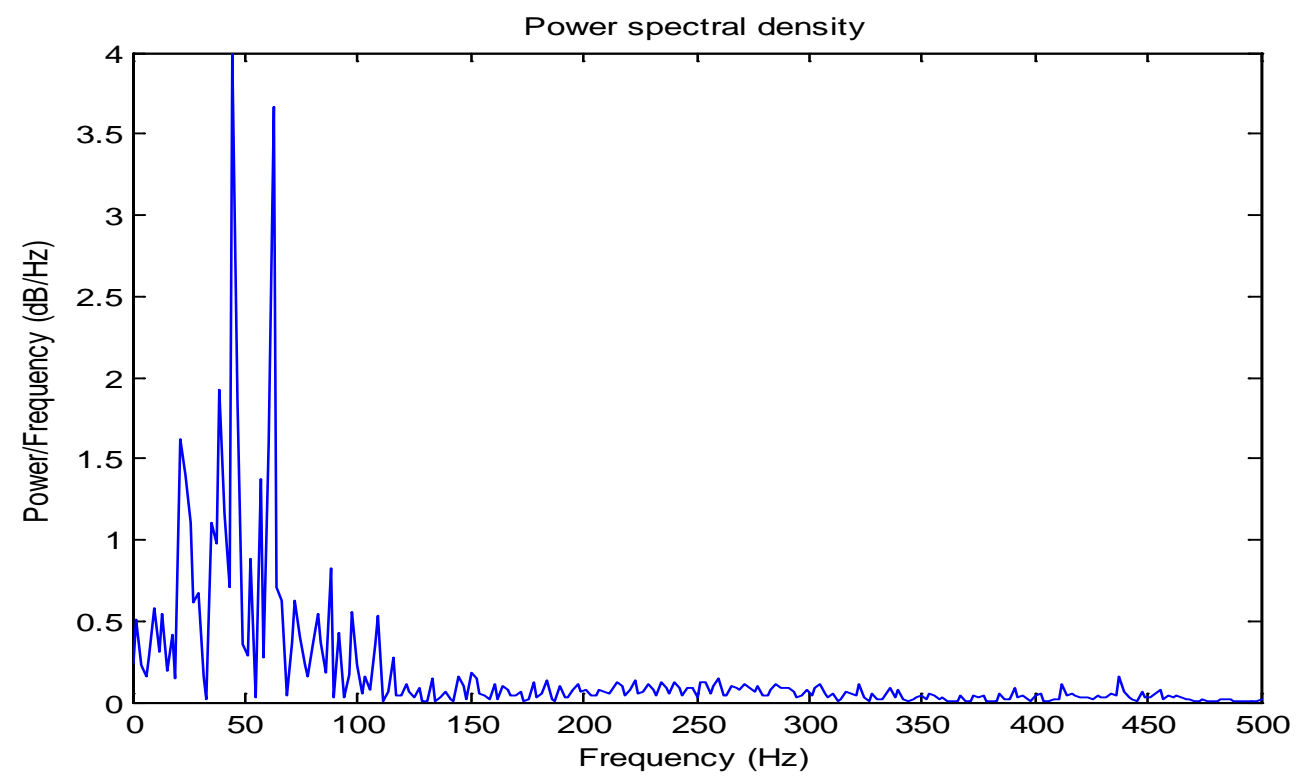

Figure 7: Power spectral density of Neuropathy EMG signal 

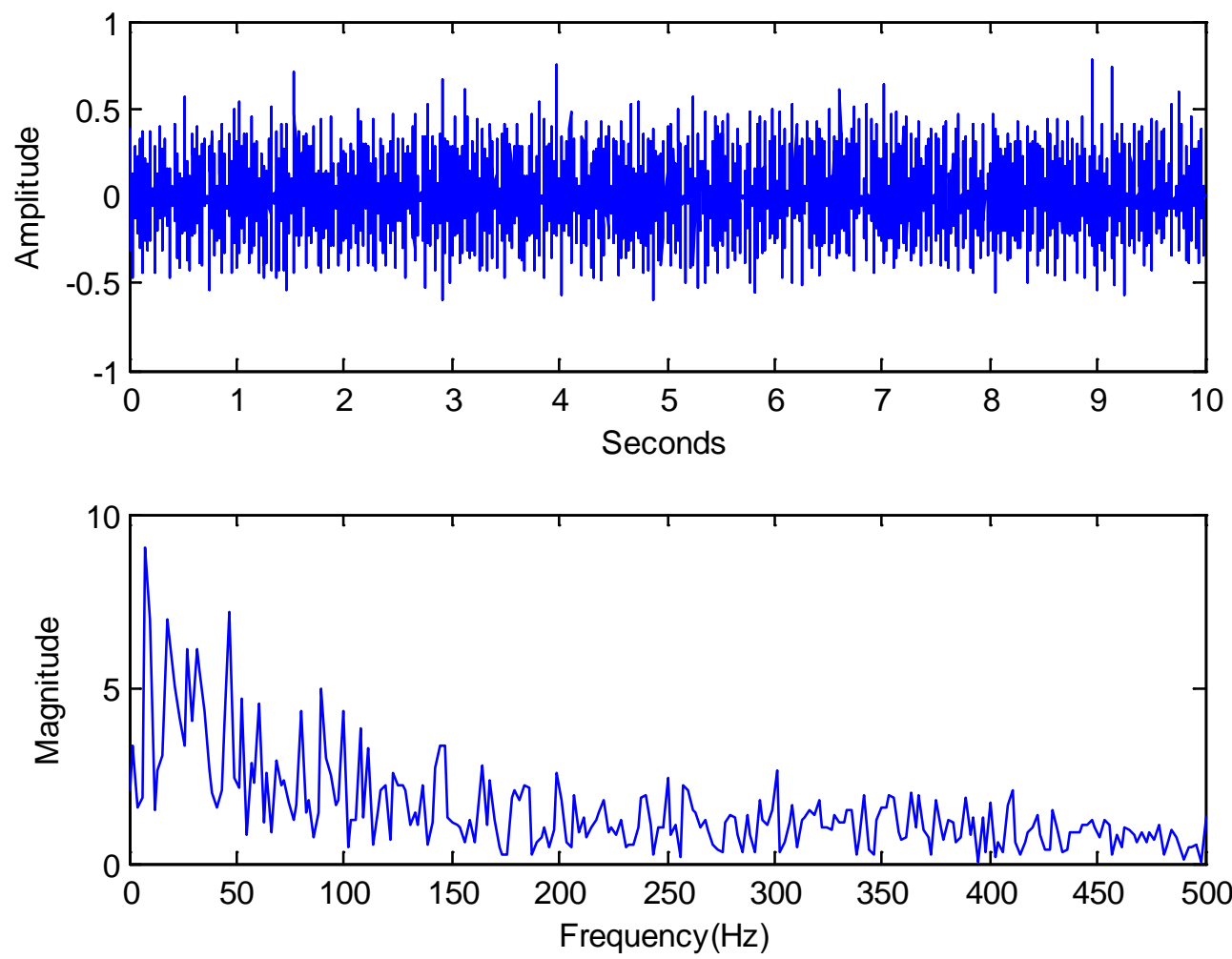

Figure 8: (a) Original Myopathy EMG signal and (b) Frequency domain representation of EMG

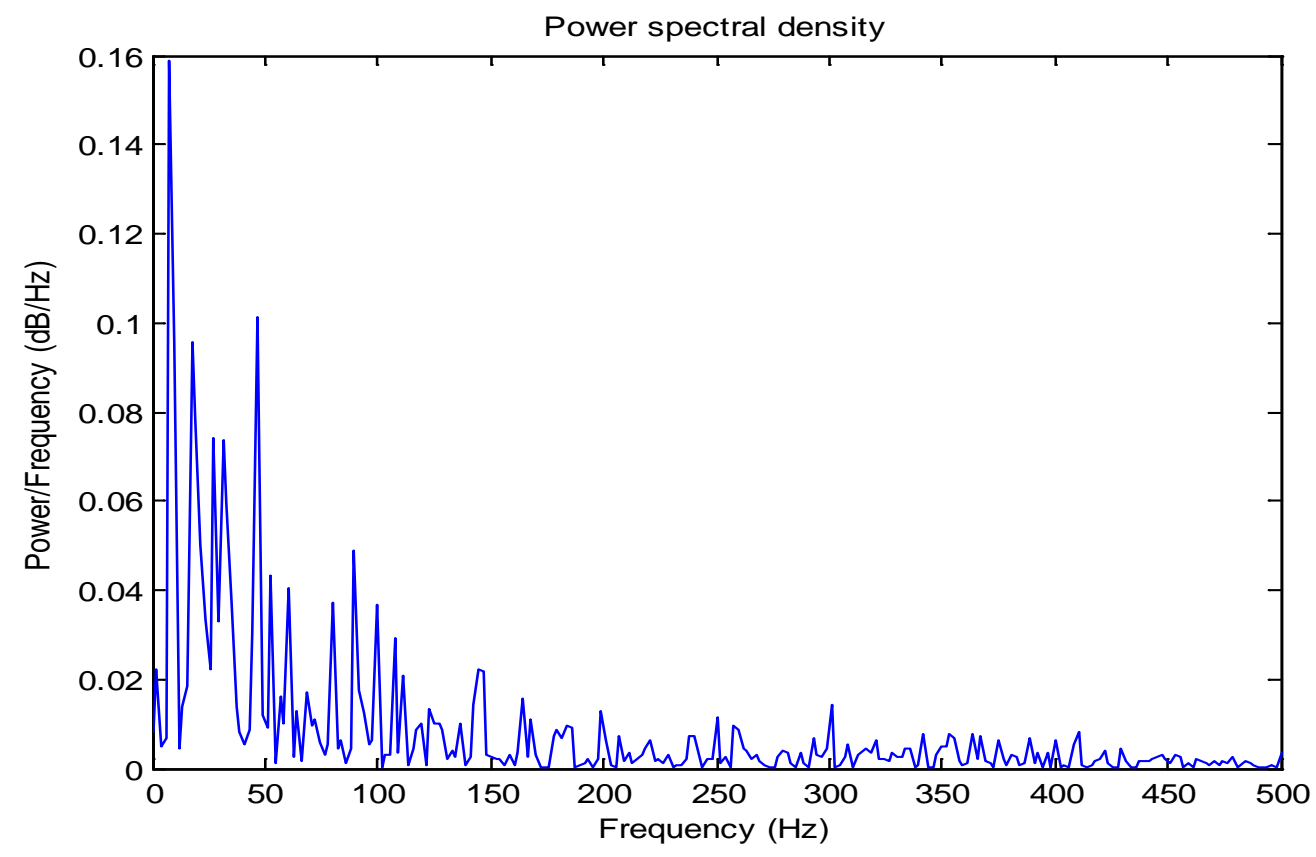

Figure 9: Power spectral density of Myopathy EMG signal

Table 1: Parameters of Different EMG signal analysis

\begin{tabular}{|c|c|c|c|}
\hline & Healthy & Myopathy & Neuropathy \\
\hline Median & 0 & -0.005 & 0.005 \\
\hline Mean Absolute Value & $4.12 \mathrm{E}-04$ & $6.92 \mathrm{E}-04$ & $2.27 \mathrm{E}-04$ \\
\hline Root Mean Square & 0.082007 & 0.096274558 & 0.23254014 \\
\hline
\end{tabular}




\begin{tabular}{|cc|c|c|c|}
\hline Average Power & 0.006725 & 0.009268791 & 0.054074917 \\
\hline Max Sower Spectrum & 0.119411 & 0.158724833 & 3.991596446 \\
\hline Magnitude & $\begin{array}{c}\text { Power Spectrum } \\
\text { Magnitude }\end{array}$ & $4.92 \mathrm{E}-07$ & $3.08 \mathrm{E}-07$ & $7.44 \mathrm{E}-04$ \\
\hline
\end{tabular}

Power spectrum of signal was examined to identify appropriate signal parameters to distinguish signal from respective patients. From power spectrum point of view, any signals can be easily analyzed and classified in terms of amplitude, in terms of power spectral density. Many parameters can be calculated to use as rule base classifier input, which is Mean Absolute Value (MAV), Average Power (AP), amplitude in terms of Root Mean Square (RMS), minimum and maximum power spectral density.

\section{Conclusion}

By decomposing of the signal it has been seen that most of the details of the signal are contained at lower scale which need less decomposition, so faster application of the wavelet. The decomposition levels depend on the length of the signal. The absence of very low and very high frequency concentration of the signal helped us to de-noise the signal from movement artifacts and external interfering noise easily. The database signal should be de-noised for detecting features properly. Extracted parameter can be used for classification of signal.

\section{References Références Referencias}

1. Mohammed Z. Al-Faiz, Abbas H. Miry, "Artificial Human Arm Driven by EMG Signal", 2012.

2. E. Criswell, Cram's "introduction to surface electromyography" Jones \& Bartlett Learning, 2010.

3. K. Englehart, et al., "Classification of the myoelectric signal using time-frequency based representations," Medical engineering \& physics, vol. 21, pp. 431438, 1999.

4. Daubechies, I., "Ten Lectures on Wavelets", SIAM, Philadelphia, 1992.

5. Grap: "An introduction to wavelets", IEEE Computing in science and Engineering, Vol: 2 (2); pp: 50-61, 1995.

6. Fahreddin Sadikoglua, Cemal Kavalcioglub, Berk Dagman, "Electromyogram (EMG) signal detection, classification of EMG signals and diagnosis of neuropathy muscle disease", 9th International Conference on Theory and application of Soft Computing, Computing with Words and Perception, 2018.

7. Farzaneh Akhavan Mahdavi1, Siti Anom Ahmad, Mohd Hamiruce Marhaban, Mohammad-R. Akbarzadeh, "Surface Electromyography Feature Extraction Based on Wavelet Transform", International Journal of Integrated Engineering, Vol. 4 No. 3, p. 1-7 2012. 